\title{
Paradigmenwechsel durch inkrementellen Wandel: Was bleibt von der Arbeitslosenversicherung?
}

\author{
Die Arbeitsmarktreformen unter dem Leitbild der Aktivierung haben zu einem Wandel \\ des Funktionszwecks der Arbeitslosenversicherung geführt. Infolge des Abbaus substan- \\ zieller Anspruchsrechte verlieren die Grundprinzipien des Versicherungssystems an Kraft, \\ es kommt zu einer Engführung des Äquivalenzprinzips und einer Unterminierung der \\ Statussicherung. Damit haben wir es mit einem Paradigmenwechsel zu tun, der auch die \\ Arbeits- und Lebensbedingungen der Kernarbeitnehmerschaft berührt, indem sich ein \\ schleichender Abschied von dem mittelschichtsorientierten Modell der sozialversiche- \\ rungsförmigen Absicherung bei Arbeitslosigkeit vollzieht.
}

SILKE BOTHFELD, PEER ROSENTHAL

\section{Einleitung}

Wenngleich die sozialpolitischen Institutionen in Deutschland eine augenscheinliche Stabilität aufweisen, haben die Reformen der vergangenen 15 Jahre das Sozialversicherungssystem doch grundlegend verändert. So unterliegt auch Deutschland wie die anderen Bismarck'schen Sozialstaaten einem grundlegenden Wandel (Palier/Martin 2007). Dabei verlaufen die Reformen inkrementell, gehen jedoch mit einem politischen Paradigmenwechsel einher. Der Bruch wird nicht sichtbar durch die Abschaffung von Institutionen, sondern vielmehr wird durch schrittweise Veränderungen eine Neudefinition des Institutionenzwecks herbeigeführt - ohne dass ein bestimmter Wendepunkt sichtbar wird (Streeck/Thelen 2005; Mahoney/ Thelen 2010).

In diesem Beitrag vertreten wir die These, dass die im Rahmen des Aktivierungsparadigmas vollzogenen Veränderungen seit 1998 und dabei insbesondere die Hartz-Reformen zu einer Schwächung der beiden Grundprinzipien des Arbeitslosensicherungssystems geführt haben. Konkret meinen wir, dass eine Engführung des Äquivalenzprinzips sowie eine Unterminierung der Statussicherung zu beobachten sind. Gleichzeitig ist es zu einer Schwächung der solidarischen und paritätischen Finanzierung gekommen.

Im Folgenden zeigen wir, an welchen Merkmalen sich Politikwandel messen lassen muss, wenn er als paradigma- tischer Wandel gelten soll (2). Anschließend stellen wir die beiden wichtigsten Grundprinzipien der Leistungsseite der Arbeitslosenversicherung, das Äquivalenzprinzip und die Statussicherung vor (3), bevor wir eine institutionelle Feinanalyse der Modifikation der Instrumente sowie ihrer Umsetzung und deren Auswirkungen zeigen. Hierbei wird deutlich, dass die Veränderung sehr kleiner Stellschrauben das System im Grundsatz verändern kann (4). Im Fazit argumentieren wir, dass die beobachtbaren Veränderungen über die Diagnose einer „Dualisierung“ des deutschen Beschäftigungssystems hinausweisen (5).

\section{Paradigmatischen Politikwandel verstehen}

Die Reichweite von Politikwandel wird vor allem durch die institutionalistischen Ansätze der Politikanalyse verständlich. Prägend für die wissenschaftliche Debatte ist hierfür vor allem die Unterscheidung von Politikwandel erster, zweiter und dritter Ordnung von Peter Hall (1993). ${ }^{1}$ Danach gelten die Anpassung von Instrumenten sowie die Einführung neuer Instrumente als unterschwelliger Wandel (erster und zweiter Ordnung), während Wandel dritter Ordnung die Neuformulierung der Politikziele meint. Bei paradigmatischem Wandel findet ein 
Wandel in allen drei Aspekten statt. Aus der Unterscheidung und dem Zusammenspiel von Veränderungen dieser drei Aspekte lassen sich wichtige Erkenntnisse zur Beschreibung und Bewertung von Wandel in der Sozialpolitik ziehen.

Erstens ist das wissenschaftstheoretische Konzept des Paradigmas, verstanden als ein für die Politik handlungsleitendes Set von normativen und kausalen Annahmen (Hall 1993), aufschlussreich für das Verständnis von Politikwandel. Demnach folgt eine Neuausrichtung von Policies, die als Varianten institutionalisierter Politikinhalte zu verstehen sind, immer auch einer neuen Definition politischer Ziele, weil neue Annahmen über Wirkungszusammenhänge oder normative Auffassungen Geltung erlangen und ältere Annahmen entwerten. Die Veränderungen im politischen Diskurs sind jedoch nicht unbedingt ursächlich, sondern begleiten meist institutionelle Reformen mit dem Ziel, die vorgenommenen Veränderungen zu legitimieren. Der politische Diskurs ist dabei eine wichtige Legitimationsressource: Politikreformen werden gerechtfertigt, ohne dass neue implizite (Verhaltens)Annahmen oder normative Festlegungen empirisch belegt oder mehrheitsfähig sein müssen. Neue diskursive Muster (hier: die Aktivierung), die mit den älteren koexistieren (Sozialversicherungsprinzip), sind somit notwendige, aber keine hinreichenden Kennzeichen für den grundlegenden Wandel von Politik. Für einen paradigmatischen Wandel bedarf es immer auch institutioneller Veränderungen. So können auch graduelle institutionelle Veränderungen, die in der Hall'schen Typologie zunächst als unterschwellig gelten - die Anpassung alter oder die Einführung neuer Instrumente - kumuliert zu einem weitreichenden Politikwandel führen (Streeck/ Thelen 2005).

Daraus folgt zweitens, dass für einen grundlegenden Politikwandel weder die „alten“ Ziele aufgegeben noch Institutionen gänzlich abgeschafft werden müssen. Tatsächlich haben empirische Analysen gezeigt, dass paradigmatische Politikwechsel selten als eine vollkommene Ablösung oder Ersetzung eines bisher gültigen durch ein neues Paradigma auftreten, sondern unterschiedliche Paradigmen nebeneinander bestehen und sich überlagern können (Streeck/Thelen 2005; Mahoney/Thelen 2010). Daher werden Regelungen innerhalb eines bestehenden Policyregimes möglicherweise uneinheitlich (inkonsistent) oder widersprüchlich (inkohärent) und es können sich unterschiedliche Praktiken gleichzeitig herausbilden (z. B. Maßnahmen der Statusgefährdung und des Statuserhalts). Inkonsistenz und Inkohärenz sind sogar typische Folgen von Politikwandel (Bothfeld 2008). Paradigmatischer Wandel lässt sich somit nicht allein an der Neudefinition von Zielen ablesen, sondern an den institutionellen Veränderungen des Policyregimes sowie den Veränderungen in der beobachtbaren Praxis, wenn diese mit einem Teil der Ziele in ein Spannungsverhältnis gerät. Streeck und Thelen zeigen mit ihrer Typologie institutionellen Wandels, dass hierzu nicht unbedingt absichtsvolle Reformen notwendig sind (Streeck/Thelen 2005): Eine Diskre- panz zwischen Policy und Praxis bzw. Politikwirkungen kann sich auch aus einer fehlenden Nachjustierung von Policies ergeben, wenn sich die Praktiken so verändern, dass sie mit den Zielen der Policies nicht mehr zusammenpassen oder sich aus dem institutionellen Geltungsbereich eines PolicyRegimes hinausentwickeln (wenn z. B. für atypisch Beschäftigte Sicherungslücken entstehen). Für diese „Verschiebung“ (Streeck/Thelen 2005) kann gleichermaßen eine Veränderung oder eine fehlende Anpassung von Policies ursächlich sein.

Drittens richtet die Hall'sche Kategorisierung von Politikwandel den Blick eben gerade auf das Zusammenwirken von materiellen und nicht-materiellen Aspekten von Politik. Die sogenannten Policy-Prinzipien vermitteln hierbei zwischen (sprachlich gefassten) Zweckbestimmungen von Regeln oder Gesetzen bzw. Normen und den materiellen institutionalisierten Regelungen (Instrumente, institutionelle Teilmechanismen). ${ }^{2}$ Die Betonung der Logiken oder Prinzipien von Politik verweist auf die politischen Steuerungsansätzen zugrunde liegenden, aber nicht immer explizit gemachten, oft nicht näher begründeten normativen Ziele von Policies sowie kausale Annahmen über das Verhältnis zwischen Instrument und Adressaten. Während eine Veränderung der Zielsetzungen oder der einzelnen Instrumente nicht notwendigerweise zu paradigmatischem Wandel führt, ist eine Veränderung der Policy-Prinzipien als ein Kristallisationspunkt grundlegenden, paradigmatischen Wandels zu verstehen.

Die Entwertung eines Grundprinzips kann zu einer Destabilisierung des Policy-Regimes im Sinne einer Unterminierung von dessen Funktionalität oder Legitimität führen. Die institutionelle Perspektive auf Politikwandel unterstreicht somit die implizite Normativität von Politik und die politische Dimension von Politikwandel, die sich sowohl im politischen Diskurs als auch in der institutionellen Ausgestaltung von Policies und bei der Herausbildung neuer Praktiken (Verhalten, Einstellungen) abzeichnet. Streeck und Thelen haben hingegen deutlich gemacht, dass paradigmatischer Wandel institutionelle und normative Veränderungen mit sich bringt, aber durchaus auch durch inkrementelle Veränderungen ausgelöst werden kann. Paradigmatischer Wandel ist damit nicht notwendiger Weise in seiner ganzen Tragweite intentional.

\footnotetext{
1 Peter Hall entwickelte diese Kategorisierung am Beispiel des wirtschaftspolitischen Wandels in Großbritannien und erklärte die Durchsetzung (neo)liberaler Politik mit der „Erschöpfung" des keynesianischen Paradigmas für die Erklärung gesamtwirtschaftlicher Zusammenhänge (Hall 1986).
}

2 Unter Policy-Prinzipien verstehen wir die institutionalisierten Grundlogiken, nach denen Sozialleistungen, v. a. Transfers, gewährt werden (s. a. Nullmeier/Rüb 1993 am Beispiel der Rentenpolitik). 


\section{Grundprinzipien der Arbeitslosen- versicherung unter dem Sozial- versicherungsparadigma}

Grundsätzlich unterscheiden sich Sozialversicherungssysteme von anderen Leistungssystemen in der Art der Sozialleistungen, der Regulierung des Zugangs zu diesen Leistungen, hinsichtlich des Finanzierungsmodus sowie im Hinblick auf die Beteiligung der Sozialpartner an der Selbstverwaltung (Bonoli/Palier 2000). Anders als in den steuerfinanzierten universalistischen Systemen ist hier der redistributive Mechanismus der vertikalen Umverteilung zwischen Groß- und Geringverdienern weniger stark ausgeprägt (Korpi/Palme 1998). Dementsprechend ist auch die Leistungsseite der deutschen Arbeitslosenversicherung durch zwei Grundprinzipien geprägt: die Leistungsgerechtigkeit, also das Äquivalenzprinzip bei der Leistungsbemessung (Nullmeier/Vobruba 1994), sowie die Garantie der Statussicherung im Erwerbsverlauf durch das transferorientierte Leistungssystem (Kaufmann 2003). Damit stellt das deutsche System ein sehr typisches, in seiner Umverteilung begrenztes, auf die Mittelschicht zielendes sowie ein hochgradig akzeptiertes (Nüchter/Schmid 2012) Sozialsystem dar.

\subsection{Das Äquivalenzprinzip in der Leistungs- bemessung}

Das den deutschen Sozialversicherungssystemen zugrunde liegende Gerechtigkeitsprinzip, die Leistungsgerechtigkeit, findet sich am deutlichsten in der Rentenversicherung, aber auch in der Arbeitslosenversicherung wieder. Auf der Basis erreichter Marktpositionen und daraus abgeleiteter Beitragszahlungen entstehen rechtsförmige Ansprüche an das Versicherungssystem. Das Äquivalenzprinzip setzt diese Gerechtigkeitsauffassung um und sorgt dafür, dass Beiträge und Leistungen in einem Entsprechungsverhältnis stehen. Die Stellschrauben, mit denen dieses Verhältnis reguliert wird, sind die Regelungen des Leistungszugangs, die Ausgestaltung der Höhe der Leistungen sowie die Festlegung der Dauer der Leistungszahlungen.

Das Äquivalenzprinzip ist durch die Unter- und Obergrenzen der Versicherungspflicht, die geringfügig entlohnte Beschäftigung auf der einen und die Beitragsbemessungsgrenze auf der anderen Seite, begrenzt. Kommt die Versicherungspflicht zur Anwendung, müssen für den Leistungszugang innerhalb eines definierten Zeitraums (Rahmenfrist) für eine festgelegte Dauer (Anwartschaftszeit) Beiträge entrichtet worden sein. Sind diese Voraussetzungen nicht erfüllt, entstehen trotz vorangegangener Beitragszahlungen keine Ansprüche. Somit gilt das Äquivalenzprinzip erst ab einem bestimmten Grad der Beschäftigung und schließt überdurchschnittliche Einkommensanteile von der Risikoabsicherung aus.

Die Höhe der Lohnersatzleistung bemisst sich an den innerhalb der Unter- und Obergrenzen einkommenspropor- tional geleisteten Beiträgen; den Beitragszahlungen stehen damit äquivalente Leistungen gegenüber. Der soziale Ausgleich der Arbeitslosenversicherung manifestiert sich damit in Form einer horizontalen Umverteilung zwischen Versicherten mit niedrigem und hohem Arbeitslosigkeitsrisiko.

Die Dauer der Beitragszahlungen bestimmt die Dauer des Leistungsbezugs und steht in einem Entsprechungsverhältnis von zwei zu eins. Es gibt eine maximale Bezugsdauer, die durch altersspezifische Regelungen ausdifferenziert ist, sodass älteren langjährigen Beitragszahlern und Beitragszahlerinnen längere Bezugsdauern gewährt werden. Die Arbeitslosenversicherung ist damit so ausgestaltet, dass Langzeitversicherte gegen das soziale Risiko Arbeitslosigkeit entsprechend ihrem vorherigen Lohneinkommen abgesichert sind, wobei der Einkommensverlust temporär und im Rahmen der Unter- und Obergrenzen beitragsäquivalent kompensiert wird.

\subsection{Die Statussicherung als Ziel sozialer Sicherung}

Das zweite Prinzip, die relative Statussicherung, ist für die Sozialstaaten Bismarck'scher Prägung typisch. Sie ergibt sich aus der Kopplung nicht nur des Beitrags, sondern auch der Leistungshöhe an das versicherte Einkommen sowie aus der Dynamisierung der Leistung entsprechend der allgemeinen Lohnentwicklung. Außerdem ist durch Kopplung der Bemessungsgrenze an das Durchschnittseinkommen die Leistungsbemessung am durchschnittlichen Lebensstandard der Mittelschicht orientiert, wobei in Deutschland - anders als in anderen Sozialversicherungssystemen wie z. B. in Frankreich oder Belgien (Bothfeld 2012) - keine Ausgleichsregelungen für Geringverdiener oder Mindestleistungsbeträge vorgesehen sind.

Darüber hinaus ist erstens die institutionelle Ausgestaltung des Reziprozitätsverhältnisses von Bedeutung. Die Reziprozitätsnormen definieren die wechselseitigen Verpflichtungsbeziehungen zwischen Individuum und Solidargemeinschaft, indem Kriterien für die Aufnahme einer Beschäftigung oder die Maßnahmeteilnahme festgelegt werden. Es geht also um die direkte Verknüpfung von Leistungsbezug, Zumutbarkeit und aktiver Arbeitsmarktpolitik. Die Grundlogik war hier, dass die Struktur und Ausrichtung der Arbeitsmarktmaßnahmen auf den Erhalt des erreichten beruflichen Status und die Förderung der Qualifikation der Arbeitslosen ausgerichtet waren. Ziel war es, den einmal erreichten Berufs- und Qualifikationsstatus und die damit verbundenen Einkommenschancen sowie den sozialen Status zu erhalten. Auch sollte die Definition dessen, was als eine zumutbare Beschäftigung verstanden wurde, nicht das erreichte Qualifikationsniveau oder das Einkommensniveau gefährden. Die institutionellen Regelungen und deren Anwendung spiegelten damit die Grundidee des Arbeitsförderungsgesetzes wider, wonach nicht nur Arbeitslosigkeit vermieden, sondern auch unterwertige Beschäftigung reduziert und Aufstiegsmobilität von Beschäftigten ausgelöst werden sollte (Lutz 1984). Eine solche Ausgestaltung 
folgte damit moralökonomisch der Vorstellung, dass der Bezug von Versicherungsleistungen aufgrund vorheriger Beitragszahlungen legitim sei; dementsprechend war (und ist) die soziale Akzeptanz des Arbeitslosenversicherungssystems hoch. Aus der ökonomischen Perspektive galten Phasen der Arbeitslosigkeit als die Effektivität steigernde „Suchphasen“, die die qualitative Passförmigkeit (matching) zwischen Arbeitsangebot und -nachfrage verbessern sollten.

Das Grundprinzip der Statussicherung galt dabei nicht nur für die Bezieherinnen und Bezieher von Arbeitslosengeld, sondern auch der Arbeitslosenhilfe als eine Leistung der Arbeitslosenversicherung. Zwar handelte es sich bei der Arbeitslosenhilfe um eine bedürftigkeitsgeprüfte und steuerfinanzierte Leistung, die unbefristet gewährt wurde. Sie orientierte sich aber in ihrer Höhe am vorherigen Einkommen, gründete - seit der vollständigen Abschaffung der „originären“ Arbeitslosenhilfe - auf dem einstigen, nunmehr erschöpften Anspruch auf Arbeitslosengeld und damit indirekt auf vorhergehenden individuellen Beitragszahlungen (Knuth 2006).

\section{Wandel der Grundprinzipien der Arbeitslosenversicherung unter dem Aktivierungsparadigma}

Inwiefern lässt sich nun also ein grundlegender Wandel der Arbeitslosenversicherung ablesen? Im Folgenden wird der Wandel der Grundprinzipien durch die mit den Reformen verbundenen Veränderungsprozesse nachgezeichnet, die sich nun am Paradigma der Aktivierung ausrichten, ohne sich jedoch vollständig von der Logik des Sozialversicherungsparadigmas zu lösen. Die Aktivierungsreformen orientieren sich in normativer Hinsicht an einem neuen Gerechtigkeitsbegriff, nachdem nicht mehr Leistungsgerechtigkeit, die soziale Statussicherung der Mittelschicht und eine solidarische und paritätische Verantwortung, sondern eine eng gefasste Norm der Reziprozität im Zentrum steht (Gronbach 2012). Die möglichst schnelle Arbeitsaufnahme wird zur zentralen Stellschraube des am individuellen Verhalten orientierten Leistungssystems. Trotz des Fortbestands der „alten“ Sozialversicherungslogik, kommt es in der Summe der institutionellen Veränderungen zu einem grundlegenden Wandel, der sich als ein „schleichender Abschied“ vom traditionellen deutschen System der Arbeitslosenversicherung und damit als Paradigmenwechsel beschreiben lässt.

\subsection{Die Engführung des Äquivalenzprinzips}

Die Engführung des Äquivalenzprinzips kommt erstens darin zum Ausdruck, dass der Zugang zur Arbeitslosenversicherung enger gefasst wurde. Die Rahmenfrist wurde zum Februar 2006 von 36 auf 24 Monate gekürzt, zur Erreichung der Mindestbezugsdauer von sechs Monaten sind damit zwölf Beitragsmonate in den letzten zwei Jahren nötig. Für die Erreichung der generellen Höchstbezugsdauer von zwölf Monaten muss eine durchgängige Beitragszahlung in den letzten 24 Monaten erfolgt sein.

Diese Veränderungen erschweren es unstetig Beschäftigten, Ansprüche auf Arbeitslosengeld zu erwerben, auch wenn sie in ihrer Gesamterwerbsbiografie langjährig in die Versicherung eingezahlt haben. Berechnungen des Instituts für Arbeitsmarkt- und Berufsforschung (IAB) belegen, dass durch die Veränderungen von Anwartschaftszeit und Rahmenfrist allein im Jahr 201050.000 Personen weniger Ansprüche auf Arbeitslosengeld gehabt haben (Jahn/Stephan 2012). Anstatt es der wachsenden Gruppe atypisch Beschäftigter zu erleichtern, Ansprüche der Arbeitslosenversicherung zu erwerben, werden durch die Engfassung des Äquivalenzprinzips Zugangshürden zu Leistungsansprüchen gerade für unstet Beschäftigte (v. a. befristet Beschäftigte und Leiharbeitnehmer), die ein höheres Arbeitslosigkeitsrisiko haben, errichtet. Von der Arbeitgeberseite wird dies teilweise mit einem Gerechtigkeitsargument begründet, das mit dem Äquivalenzprinzip nicht vereinbar ist: Obgleich diese auch in die Versicherung einbezahlt haben, wird kurzfristig Beschäftigten - unabhängig von ihrer beruflichen Qualifikation und der Höhe ihrer vorgehenden Erwerbseinkommen - ein Anreizproblem hinsichtlich der zügigen Aufnahme einer neuen Beschäftigung unterstellt, wenn ihr Status durch Zugang zum Arbeitslosengeldbezug kurzfristig gesichert ist. Die Folgen der arbeitsrechtlichen Deregulierung werden somit externalisiert, da das erhöhte Arbeitslosigkeitsrisiko individualisiert wird. Daran wird deutlich, dass Gerechtigkeitsvorstellungen in vielen Fällen direkt mit impliziten Verhaltensannahmen (hier: moral hazard) gerechtfertigt werden. Allerdings erfolgt diese Zuschreibung selektiv, wodurch unterschiedliche soziale Gruppen definiert werden, die einen unterschiedlichen Grad sozialen Schutzes genießen. Als legitime Leistungsbezieher gelten in dieser Sichtweise allein langjährig kontinuierlich Versicherte und Normalbeschäftigte, deren Verhaltensmodell zum Referenzmodell erhoben wird.

Allerdings wurde 2009 eine bis Ende 2014 befristete Sonderregelung für kurzfristig Beschäftigte eingeführt, mit der dieses Problem eingeschränkt werden soll. Sie sieht eine verkürzte Anwartschaftszeit von sechs Monaten vor, wenn sich die in der Rahmenfrist zurückgelegten Beschäftigungstage überwiegend aus versicherungspflichtigen Beschäftigungen ergeben, die auf nicht mehr als zehn Wochen (vor Juli 2012 sechs Wochen) im Voraus durch Arbeitsvertrag zeit- oder zweckbefristet sind, und das in den letzten zwölf Monaten vor der Beschäftigungslosigkeit erzielte Arbeitsentgelt die zum Zeitpunkt der Anspruchsentstehung maßgebliche Bezugsgröße nicht übersteigt. ${ }^{3}$ Sie erwies sich in ihrer Wirkung aber als

3 Im Jahr 2013 lag die Bezugsgröße bei $32.240 €$ in West- und $27.300 €$ in Ostdeutschland. 
begrenzt (Werner et al. 2012). Eine zweite Tendenz besteht in der Ausweitung des Zugangs zur Arbeitslosenversicherung für zuvor arbeitslose Selbstständige, die sich seit 2006 unter bestimmten Voraussetzungen freiwillig versichern können.

Allerdings erhält ein hoher Anteil an Personen wegen vorangegangener Niedriglohnbeschäftigung nur geringe Leistungen aus der Arbeitslosenversicherung und zusätzlich ergänzende Leistungen der Grundsicherung, um das Existenzminimum der jeweiligen Haushalte zu sichern - im Jahr 2011 traf dies auf gut ein Viertel aller Neueintritte zu (BA 2011). Rund ein Drittel der Parallelbezieher und -bezieherinnen von Arbeitslosengeld I und Arbeitslosengeld II (Aufstocker) war zuvor in Leiharbeit tätig (BA 2011). Zwar entspricht hier die Höhe des Arbeitslosengeldes I dem Äquivalenzprinzip der Versicherung. Zudem war die das Existenzminimum nicht erreichende Statussicherung auch in der Arbeitslosenhilfe ein nicht selten anzutreffendes Phänomen. Unstetigkeit und Niedriglohn treten aber häufig zusammen auf, und mit steigendem Anteil derartiger Beschäftigungsverhältnisse wächst dieses Problem.

Trotz der Sonderregelung für kurzfristig Beschäftigte werden in wachsendem Maße Versicherungsbeiträge geleistet, aus denen keine oder keine bedarfsdeckenden Leistungsansprüche erwachsen. Die oftmals vertretene Annahme, dass ein Übergang in die Grundsicherung erst nach Auslaufen eines Arbeitslosengeld-Anspruchs stattfände, wird somit relativiert (Fuchs 2012). Zudem folgt für diesen Personenkreis nicht nur kein (bedarfsdeckender) Leistungsanspruch aus dem Versicherungssystem, sondern die Aufstocker sind infolge der Zuordnung zum Fürsorge- und nicht zum Versicherungssystem auch mit dem strengeren Reziprozitätsregime der Grundsicherung konfrontiert.

Hinzu kommt, dass öffentlich geförderte sozialversicherungspflichtige Beschäftigung nicht mehr der Arbeitslosenversicherungspflicht unterliegt und somit Beschäftigte im „Zweiten Arbeitsmarkt“ keine Leistungsansprüche an die Arbeitslosenversicherung mehr aufbauen können (Rosenthal 2012).

Die Engführung des Äquivalenzprinzips spiegelt sich auch in der Verkürzung der Bezugsdauer wider, womit Leistungsansprüche auch von Normalbeschäftigten beschnitten wurden. Seit Februar 2006 waren selbst für langjährige Beitragszahler und -innen über 55 Jahre nur noch 18 Monate Leistungsbezug möglich. Diese Regelung hatte allerdings nur bis Januar 2008 Bestand, bevor nach langer und intensiver Diskussion der Leistungsanspruch für 50- bis 54-Jährige erneut auf 15 Monate und für über 58-Jährige auf 24 Monate angehoben wurde. Im Ergebnis sind aber über 45-jährige Arbeitslose schlechter gestellt als vor 2006. Mit der kürzeren maximalen Bezugsdauer entfällt zumindest teilweise die Anerkennung langjähriger Beitragszahlung als eine Art „Lebensleistung“ und führt zu einer Verschiebung der Leistungsgerechtigkeit. ${ }^{4}$

Insgesamt betrachtet erfüllt die Arbeitslosenversicherung für einen immer geringeren Anteil der Beschäftigten das Ziel, beim Eintritt von Arbeitslosigkeit den Einkom- mensausfall zu kompensieren: Während im Jahr 1999 noch $40 \%$ der Arbeitslosen Arbeitslosengeld bezogen, waren es 2011 nur noch ein Viertel, wenngleich dieser Trend zwischen 2008 und 2009 aufgrund der Auswirkungen der Wirtschaftskrise kurzfristig unterbrochen wurde (Rosenthal 2012). Das Äquivalenzprinzip wird damit in der Summe enger gefasst als zuvor und das Verhältnis zwischen Beitragsleistung und Gegenleistung durch Versicherungsansprüche neu definiert.

\subsection{Unterminierung der Statussicherung}

Die Reziprozitätsnormen wurden im Rahmen der Aktvierungsreformen mit dem Ziel novelliert, dass Arbeitslose durch eine erhöhte Konzessionsbereitschaft schneller eine Wiederbeschäftigung aufnehmen. So wurde bereits 1998 der Qualifikationsschutz aufgehoben und nach der heutigen Rechtslage gilt lediglich ein gestufter Einkommensschutz. Im Zuge der Hartz-Reformen sollte die regionale Mobilität erhöht werden, indem für Arbeitslose ohne familiäre Bindungen von Beginn der Arbeitslosigkeit an auch eine Vermittlung außerhalb des zumutbaren Pendelbereichs als zumutbar gilt. Die Verschärfungen der Zumutbarkeitsregeln wurden durch sukzessive Veränderungen im Sperrzeitenrecht (Umkehr der Beweislast und Ausdifferenzierung der Sperrzeitendauer, umgehende und frühzeitige Arbeitslosmeldung) begleitet. Auffällig ist, dass Meldeversäumnisse und verspätete Meldungen als arbeitsuchend in den vergangenen Jahren zu den wichtigsten Gründen für die Verhängung von Sperrzeiten geworden sind. Alle Sachverhalte zusammengefasst, ist die Sperrzeitquote ${ }^{5}$ von $9 \%$ im Jahr 2004 auf knapp 29 \% im Jahr 2011 gestiegen (Sozialpolitik aktuell 2012). Im Vergleich der OECD-Länder gehören die abgeforderten Verpflichtungen (Verfügbarkeit, regionale und berufliche Mobilität) in Deutschland heute zusammen mit Dänemark und Norwegen zu den strengsten Vorschriften (Venn 2012, S. 17).

Tatsächlich sind die durchschnittliche Dauer von Arbeitslosigkeitsperioden - und damit auch die Kosten von Arbeitslosigkeit - gesunken. So konnte die „Matchingeffizienz“ - also die Schnelligkeit von Stellenbesetzungsprozessen - gesteigert werden (Klinger et al. 2013). Allerdings ging dies auf Kosten der qualitativen Passförmigkeit der Wiederbeschäftigung. So ist unter anderem die Konzessionsbereitschaft arbeitsloser Bewerberinnen und Bewerber in Hinblick auf Lohnhö-

\footnotetext{
4 Die sogenannte 58er-Regelung sah bis zum 31.12.2007 vor, dass Leistungsempfänger, die das 58 . Lebensjahr vollendet hatten, gegenüber der Arbeitsverwaltung erklären konnten, dem Arbeitsmarkt nicht mehr zur Verfügung zu stehen, und dass sie trotzdem weiterhin Leistungen beziehen konnten.

5 Für das SGB III kann eine Sperrzeitquote errechnet werden, welche die Zahl der Sperrzeiten auf die Zugänge an Leistungsempfängern (Arbeitslosengeld bei Arbeitslosigkeit) bezieht (Sozialpolitik aktuell 2012).
} 
he, Arbeitsbedingungen und Qualifikationsniveau der Arbeitsplätze gestiegen (Kettner/Rebien 2007). Von den verschärften Zumutbarkeits- und Sanktionsregelungen gehen sowohl ex-post (WZB/infas 2006) als auch ex-ante (Hofmann 2012) Effekte hinsichtlich eines schnelleren Übergangs von Arbeitslosigkeit in Beschäftigung aus - auch hier um den Preis teilweise deutlicher Lohneinbußen beim Übergang von Arbeitslosigkeit in Beschäftigung (Jaenichen/Rothe in diesem Heft) sowie erhöhter Übergänge sanktionierter Arbeitsloser in Nichterwerbstätigkeit oder geringfügige Beschäftigung (Hofmann 2012). Auch hat das prominente Beispiel der ehemaligen Schlecker-Beschäftigten verdeutlicht, dass das institutionell verschärfte Zumutbarkeitsrecht in der Vermittlungspraxis weiter zugespitzt wird und das Spannungsverhältnis zwischen schneller Vermittlung und Statussicherung zulasten letzterer aufgelöst wird (Ramos Lobato et al. 2013). Die Kehrseite gesteigerter „Effizienz“ ist somit ein qualitativ schlechteres Matching, da das neue System keine ausreichende Suchphase für qualifikatorische Matchingprozesse einräumt - darauf deutet zumindest die Zunahme unterwertiger Beschäftigung hin (Hall 2010).

Die Marginalisierung des Grundprinzips der Statussicherung zeigt sich auch in der Zielbestimmung des SGB III und in der Ausgestaltung der Förderinstrumente. So definiert das Gesetz zwar immer noch, dass die Beschäftigungsstruktur ständig verbessert werden soll und unterwertiger Beschäftigung entgegenzuwirken ist. Dies wird allerdings durch den Vermittlungsvorrang konterkariert, nach dem eine Förderung nur dann als legitim gilt, wenn sie für eine dauerhafte Eingliederung erforderlich ist. Damit institutionalisiert der Vermittlungsvorrang die „work-first“-Strategie aktivierender Arbeitsmarktpolitik. Davon ist auch das „Herzstück“ (Oschmiansky 2010, S. 18) der aktiven Arbeitsmarktpolitik, die Förderung beruflicher Weiterbildung, (FbW) betroffen. Die Zahl der Neueintritte hat sich zwischen 2002 und 2004 mehr als halbiert, der Tiefstand wurde im Jahr 2005 erreicht. Zwischen 2006 und 2009 gab es einen deutlichen Anstieg der Eintritte, 2011 gingen die Eintrittszahlen fast auf das Niveau von 2006 zurück. Die Relation von Maßnahme-Eintritten und Bestand verweist auf eine strukturelle Verschiebung: Die durchschnittliche Maßnahmedauer ist zwischen 2002 und 2005 stark zurückgegangen. Die Entwicklung am aktuellen Rand zeigt, dass die Jahre zwischen 2006 und 2009 auch bezüglich der Dauer der Maßnahmen nur ein kurzes Zwischenspiel gewesen sind. Von der verkürzten Maßnahmedauer waren insbesondere die längerfristigen Umschulungsmaßnahmen betroffen, die nur noch einen verschwindend geringen Teil ausmachen (Bosch 2012). Die Entwicklung verweist darauf, dass das Spannungsverhältnis zwischen (abschlussbezogener) Qualifikationsvermittlung und schneller Arbeitsmarktintegration zugunsten des Vermittlungsvorrangs aufgelöst wurde.

Hinzu kommt, dass die Arbeitsförderung nun vornehmlich auf die Bearbeitung des Übergangs von Arbeitslosigkeit in Erwerbstätigkeit zielt und die Beschäftigtenförderung fast vollständig aus dem Blick geraten ist - so wird unter- wertige Beschäftigung nicht vermieden und Potenziale für Aufstiege am Arbeitsmarkt werden nicht ausgeschöpft (Knuth 2011). Damit fungiert die berufliche Weiterbildung infolge der Reformen und ihrer Implementation inzwischen mehr als „kurzfristige Vermittlungshilfe“ (Bosch 2012) denn als Instrument von Statussicherung und Aufstiegsmobilität. Ob intendiert oder nicht, der neue Fokus auf die Effizienz arbeitsmarktpolitischer Maßnahmen unterminiert das Ziel der Statussicherung und stellt somit die zentrale Säule des deutschen Sicherungssystems infrage.

Das Ziel der Statussicherung ist durch die Abschaffung der Arbeitslosenhilfe zusätzlich abgewertet worden, da die Arbeitslosenhilfe statussichernd ausgestaltet war und damit zumindest für den Personenkreis mit zuvor ausreichend hohem Einkommen oberhalb der Grundsicherung lag. Ein Teil der ehemaligen Bezieher von Arbeitslosenhilfe wurde aber nicht nur aus dem Versicherungs- in das neue Fürsorgesystem des SGB II ausgesteuert, vielmehr haben 200.000 ehemalige Arbeitslosenhilfe-Bezieher ihren Leistungsanspruch komplett verloren. Für einen weiteren Teil war der Übergang ins SGB II mit Leistungseinbußen verbunden (Bruckmeier/Schnitzlein 2009). Ein deutlicher Einschnitt zeigt sich auch daran, dass ein Fünftel der Neueintritte in den Arbeitslosengeld-II-Bezug zunächst vorhandenes Vermögen und Ersparnisse aufbrauchen muss, bevor die Bedürftigkeit anerkannt wird und Leistungen der Grundsicherung gewährt werden (Fuchs 2012). Bezogen bis 2004 noch über zwei Drittel der Leistungsbezieher die am vorangegangenen Einkommen orientierten Leistungen Arbeitslosengeld oder Arbeitslosenhilfe, ist dieser Anteil nach 2005 deutlich zurückgegangen und lag 2011 nur noch bei $15 \%$.

Zum 01.01.2011 wurde schließlich auch der 2005 eingeführte, jedoch befristete Zuschlag beim Übergang vom Versicherungssystem ins Fürsorgesystem gestrichen und damit das letzte Element der Statussicherung im SGB II abgeschafft. Die Reduzierung (2007) und schlussendliche Streichung (2011) der Rentenversicherungsbeiträge für SGB-II-Bezieher verdeutlichen die vollzogene Abkehr von statussichernden Elementen für Fürsorgeempfängerinnen und -empfänger.

Die Abschaffung der Arbeitslosenhilfe hat die soziale Sicherheit vom Arbeitsmarktstatus entkoppelt (Knuth 2006), da der soziale Status, der sich aus der beruflichen Stellung und der Einkommensposition einer Person ableitet, über eine Phase der Arbeitslosigkeit mit immer geringerer Wahrscheinlichkeit erhalten bleibt. Dies liegt nicht zuletzt an der Ausweitung der Reziprozitätsnormen der Fürsorge auf alle Arbeitslosen, die keine existenzsichernden Leistungen aus der Arbeitslosenversicherung erhalten. Dazu gehören wesentlich auch die Regeln zur Anrechnung von Einkommen und Vermögen auf eventuelle Ansprüche auf Grundsicherungsleistungen.

Die Antizipation der neuen Option des sozialen Abstiegs durch Arbeitslosigkeit führt nicht nur zur Entwicklung „neuer sozialer Unsicherheit“, sondern entfaltet gleichzeitig auch eine disziplinierende Wirkung auf regulär Beschäftigte (Dörre 2006). Die veränderten institutionellen Regelun- 
gen zur Zumutbarkeit und des Sperrzeitenrechts verweisen auf das Ziel, die räumliche und sektorale Mobilität von Arbeitslosen in höherem Umfang einzufordern. Die „workfirst"-Orientierung reduziert die Arbeitslosenversicherung auf die Funktion einer schnellen Vermittlungsunterstützung und wertet die Ziele der Statussicherung, Qualifikationsförderung und Aufstiegsmobilität ab, sodass sich tendenziell eine „Nivellierung von Lebenslagen auf einem Mindestsicherungsniveau" (Bothfeld et al. 2012, S. 27) abzeichnet.

Eine Ausnahme von dieser übergreifenden Tendenz bildet allerdings die Kurzarbeiterregelung, die 2008 und 2009 nach dem Eintreten der Finanzkrise und dem vorübergehenden Einbruch der Exportwirtschaft ausgeweitet wurde und der breiten Abfederung des plötzlichen Beschäftigungseinbruchs diente (Bogedan 2012). Dies verdeutlicht ebenso wie die politische Willensbekundung, auch in zukünftigen Krisen auf das Kurzarbeitergeld zurückgreifen zu wollen, dass Elemente der Statussicherung für die Kernbeschäftigten durchaus erhalten werden. Allerdings vermindert dies nicht die zunehmende Abkopplung diskontinuierlich Beschäftigter und damit auch nicht die grundsätzliche Unterminierung des Sicherheitsversprechens, das soziale Risiko Arbeitslosigkeit nach dem Prinzip der Statussicherung zu flankieren.

\section{Fazit}

Schon die Analyse der quantitativen Veränderungen im deutschen System der Arbeitslosensicherung macht deutlich, dass die Grundprinzipien der Arbeitslosenversicherung an Kraft verlieren. Aber vor allem die institutionellen Veränderungen in der Arbeitslosenversicherung erweisen sich in ihrer $\mathrm{Zu}$ sammenschau als Etappen paradigmatischen Wandels. Es verbinden sich Elemente des „,alten“ Sozialversicherungs- mit Elementen des „neuen“ Aktivierungsparadigmas. Diese Verbindung führt dazu, dass sich der Institutionenzweck verändert, indem die in der Arbeitslosenversicherung angelegten Spannungsverhältnisse neu gewichtet werden. Die Institution wird somit zwar nicht abgeschafft, aber neu ausgerichtet. Diese Veränderungen sehen wir bei der Sicherung bei Arbeitslosigkeit darin, dass die Dauer des Leistungsbezugs reduziert, die Arbeitslosenhilfe als lohnbezogene Leistung des Arbeitslosensicherungssystems abgeschafft und die Zugänge zum Versicherungssystem enger gefasst worden sind. Zudem sind die Reziprozitätsnormen - also das Zumutbarkeits- und Sperrzeitenrecht - strenger gefasst worden. Die Veränderungen bei der Ausgestaltung und Nutzung des einstigen Herzstücks aktiver Arbeitsmarktpolitik - der Förderung beruflicher Weiterbildung - bilden einen weiteren Baustein im Abschied von zentralen Grundprinzipien und Zielen der Arbeitslosenversicherung. Begleitend hierzu wird das Grundprinzip der paritätischen und staatlich garantierten Finanzierung sukzessive durch die Absenkung der Beiträge und die Abschaffung des Bundeszuschusses infrage gestellt und in Konsequenz der Verlust der arbeitsmarktpolitischen Gestaltungsmacht der Bundesagentur für Arbeit als selbstverwaltete Sozialversicherung (Klenk 2012) verschärft.

In diesem Sinne führt inkrementeller Wandel durch die spezifische Modifikation von Instrumenten und ihrer Umsetzung zu einer Neugewichtung der institutionellen Grundprinzipien. Infolge des skizzierten Wandels kommt es zu einer Abschwächung des Äquivalenzprinzips, der Statussicherung und des Solidarprinzips. Damit geht der Wandel des Sicherungssystems bei Arbeitslosigkeit über den Befund einer zunehmenden Dualisierung (Palier/Thelen 2010; Dingeldey 2010) hinaus: Der Begriff der Dualisierung verweist lediglich auf eine sinkende Deckungsquote der Sozialversicherungen und eine stärkere Gewichtung von Fürsorgesystemen und damit auf eine zunehmende - auch institutionelle - Spaltung zwischen Kern und Peripherie. Und er betrachtet die Sozialversicherungen gleichzeitig als stabil. Wir dagegen konstatieren auch einen Wandel der Kerninstitution selbst. Anders gesagt: Neben einer Dualisierung haben wir es mit einem Wandel der Arbeitslosenversicherung zu tun, der auch die Arbeits- und Lebensbedingungen der Kernarbeitnehmerschaft berührt (Betzelt/Bothfeld 2011).

Der Umbau der Arbeitslosenversicherung in den Kernaspekten ihres Leistungssystems ist gleichbedeutend mit der Abkehr von einem, wenn nicht dem zentralen Ziel der auf die Statussicherung orientierten Sozialversicherung: dem Schutz und Erhalt von sozialen Anspruchsrechten der Mittelschicht. Dadurch, dass der Schutz des sozialen Status als Sicherungsziel in zentralen Punkten durch den Vorrang der möglichst schnellen Wiederbeschäftigung ersetzt wird und im Falle fehlender Existenzsicherung durch Leistungen der Arbeitslosenversicherung die Reziprozitätsnormen der Grundsicherung Anwendung finden, rückt die Fürsorgelogik für einen großen Teil der Arbeitslosen an die Stelle der Sozialversicherungslogik. Das Problem der Unterminierung der Statussicherung liegt darin, dass mit dem sozialen Status, der sich in einer Erwerbsgesellschaft aus dem beruflichem Status, der Arbeitsmarkt- und Einkommensposition speist, substanzielle soziale Rechte verknüpft sind, die mit der Zugehörigkeit zur Mittelschicht einhergehen. Der Abbau der Statussicherung bedeutet daher auch einen Abbau sozialer Bürgerrechte.

Pragmatische Ansatzpunkte für die Revitalisierung der Grundprinzipien der Arbeitslosenversicherung wären erleichterte Ansprüche auf und eine verlängerte Bezugsdauer von Arbeitslosengeld, eine (reflexive) Regulierung des Arbeitsmarkts, die die Risiken unsteter Beschäftigungsformen nicht einseitig auf die Beschäftigten verschiebt, sondern diese ebenso vor den Risiken des Marktes schützt, eine Neudefinition der Zumutbarkeitsregeln sowie die Neustrukturierung der Arbeitsförderung. Dies könnte als ein Ausgangspunkt für die Wiederaufnahme konzeptioneller Überlegungen für eine Weiterentwicklung der Arbeitslosen- zu einer inklusiven und präventiv ausgerichteten Arbeitsversicherung und damit der Stärkung sozialer Bürgerrechte genutzt werden. 


\section{LITERATUR}

Betzelt, S./Bothfeld, S. (2011): The erosion of social status: The case of Germany, in: Betzelt, S./Bothfeld, S. (Hrsg.): Activation and labor market reforms in Europe. Challenges to social citizenship, Basingstoke, S. 103-124 Bogedan, C. (2012): Arbeitsmarktpolitik im Spiegel konjunktureller Entwicklungen: Die politisch motivierte unterschiedliche Nutzung des Kurzarbeitergeldes 1973-2010, in: Bothfeld, S./Sesselmeier, W./Bogedan, C. (Hrsg.), a.a. O.,

S. $126-142$

Bonoli, G./Palier, B. (2000): How do welfare states change?, in: European Review 8 (3), S. 333-352

Bosch, G. (2012): Berufliche Weiterbildung in Deutschland 1969 bis 2009: Entwicklung und Reformoptionen, in: Bothfeld, S./Sesselmeier, W./Bogedan, C. (Hrsg.), a. a. O., S. 106-125

Bothfeld, S. (2008): Under reconstruction: Die Fragmentierung des deutschen Geschlechterregimes durch die neue Familienpolitik, ZeS-Arbeitspapier 1/2008, Bremen

Bothfeld, S. (2012): Kindererziehung und Pflegezeiten: Wie anpassungsfähig sind die Sozialversicherungssysteme? Deutschland im internationalen Vergleich, ZeS-Arbeitspapier 3/2012, Bremen

Bothfeld, S./Sesselmeier, W./Bogedan, C. (2012): Arbeitsmarktpolitik - ein emanzipatorisches Projekt in der sozialen Marktwirtschaft, in: Dies. (Hrsg.), a. a. O., S. $338-350$

Bothfeld, S./Sesselmeier W./Bogedan, C. (2012) (Hrsg.): Arbeitsmarktpolitik in der Sozialen Marktwirtschaft. Vom Arbeitsförderungsgesetz zum Sozialgesetzbuch II und III, Wiesbaden

Bruckmeier, K./Schnitzlein, D. (2009): Der Übergang von Arbeitslosenhilfeempfängern in das SGB II. Eine empirische Analyse anhand von Befragungsdaten, in: Sozialer Fortschritt 58 (1), S. 1-9

Bundesagentur für Arbeit (BA) (2011): Unmittelbare Zugänge aus Beschäftigung in Arbeitslosigkeit SGB II, Nürnberg

Dingeldey, I. (2010): Agenda 2010: Dualisierung der Arbeitsmarktpolitik, in: Aus Politik und Zeitgeschichte 60 (48), S. 18-25

Dörre, K. (2006): Prekäre Arbeit: unsichere Beschäftigungsverhältnisse und ihre sozialen Folgen, in: Arbeit 15 (3), S. 181-193

Fuchs, B. (2012): Gründe für den Arbeitslosengeld-II-Bezug: Wege in die Grundsicherung, IAB-Kurzbericht (25), Nürnberg

Gronbach, S. (2012): Soziale Gerechtigkeitsleitbilder in der Arbeitsmarktpolitik - von der Verteilung zurTeilhabe, in: Bothfeld, S./Sesselmeier, W./Bogedan, C. (Hrsg.), a. a. O., S. 43-56

Hall, P. A. (1986): Governing the economy: The politics of state intervention in Britain and France, New York

Hall, P. A. (1993): Policy paradigms, social learning, and the state, in: Comparative Politics 25 (3), S. $275-296$

Hall, A. (2010): Arbeiten Frauen und Männer unter ihrem Wert? Zum Einfluss dualer und schulischer Ausbildungsberufe auf ausbildungsinadäquate Erwerbstätigkeit in Deutschland, in: Schweizerische Zeitschrift für Soziologie 36 (1), S. 131-159

Hofmann, B. (2012): Short- and long-term ex-post effects of unemployment in surance sanctions: Evidence from West Germany, in: Jahrbücher für Nationalökonomie und Statistik 232 (1), S. 31-60

Jahn, E./Stephan, G. (2012): Leistungsansprüche bei kurzen Beschäftigungszeiten: Arbeitslosengeld - wie lange man dafür arbeiten muss, IAB-Kurzbericht (19), Nürnberg

Kaufmann, F.-X. (2003): Sicherheit: Das Leitbild beherrschbarer Komplexität, in: Lessenich, S. (Hrsg.): Wohlfahrtsstaatliche Grundbegriffe. Historische und aktuelle Diskurse, Frankfurt a. M./New York, S. 73-104

Kettner, A./Rebien, M. (2007): Hartz-IV-Reform: Impulse für den Arbeitsmarkt, IAB-Kurzbericht (19), Nürnberg

Klenk, T. (2012): Vom Arbeitsförderungsgesetz zum Sozialgesetzbuch II und III: Wandel der korporatistischen Arbeitsverwaltung?, in: Bothfeld, S./Sesselmeier W./Bogedan, C. (Hrsg.), a. a. O., S. 276-290

Klinger, S./Rothe, T./Weber, E. (2013): Makroökonomische Perspektive auf die Hartz-Reformen: Die Vorteile überwiegen, IAB-Kurzbericht (11), Nürnberg Knuth, M. (2006): „Hartz IV“ - die unbegriffene Reform, in: Sozialer Fortschritt 55 (7), S. $160-168$

Knuth, M. (2011): Widersprüchliche Dynamiken im deutschen Arbeitsmarkt, in: WSI-Mitteilungen 64 (11), S. 580-587, http://www.boeckler.de/ wsi_38168_38177.htm
Korpi, W./Palme, J. (1998): The paradox of redistribution and strategies of equality: Welfare state institutions, inequality, and poverty in the Western countries, in: American Sociological Review 63 (5), S. 661-687

Lutz, B. (1984): Der kurze Traum immerwährender Prosperität: eine Neuinterpretation der industriell-kapitalistischen Entwicklung im Europa des 20. Jahrhunderts, Frankfurt a. M./New York

Mahoney, J./Thelen, K. (Hrsg.) (2010): Explaining institutional change: Ambiguity, agency, and power, Cambridge

Nullmeier, F./Rüb, F. W. (1993): DieTransformation der Sozialpolitik. Vom Sozialstaat zum Sicherungsstaat, Frankfurt a. M./New York

Nullmeier, F./Vobruba, G. (1994): Gerechtigkeit im sozialpolitischen Diskurs, in: Döring, D./Nullmeier, F./Pioch, R./Vobruba, G. (Hrsg.): Gerechtigkeit im Wohlfahrtsstaat, Marburg, S. 9-66

Nüchter, O./Schmid, A. (2012): Eine subjektive Dimension der Arbeitsmarktpo litik. Einstellungen zur Arbeitslosenversicherung und Grundsicherung für Arbeitsuchende in Deutschland, in: Bothfeld, S./Sesselmeier, W./Bogedan, C. (Hrsg.), a. a. O., S. 160-176

Oschmiansky, F. (2010): Aktive Arbeitsförderinstrumente seit Einführung des SGB III: Rückblick und Ausblick, in: Sozialer Fortschritt 59 (1), S. 16-23

Palier, B./Martin, C. (2007): Editorial Introduction. From „a frozen Landscape“ to structural reforms:The sequential transformation of Bismarckian welfare systems, in: Social Policy \& Administration 41 (6), S. 535-554

Palier, B./Thelen, K. (2010): Institutionalizing dualism. Complementarities and change in France and Germany, in: Politics \& Society 38 (1), S. 119-148 Ramos Lobato, P./Zahradnik, F./Dietz, M./Knapp, B./Sprenger, C. (2013): Vermittlung ehemaliger Schlecker-Beschäftigter: Nicht nur eine Frage der Motivation, IAB-Kurzbericht (7), Nürnberg

Rosenthal, P. (2012): Der Wandel der Arbeitslosenversicherung: Selektivität, Einkommenssicherung und Reziprozitätsnormen von 1969 bis heute, in: Bothfeld, S./Sesselmeier, W./Bogedan C. (Hrsg.), a. a. O., S. 143-159 Schmid, G. (2002): Wege in eine neue Vollbeschäftigung: Übergangsarbeitsmärkte und aktivierende Arbeitsmarktpolitik, Frankfurt a. M./New York Sozialpolitik aktuell (2012): Sperrzeitquote der EmpfängerInnen von Arbeits losengeld I 2004-3/2012

Streeck, W./Thelen, K. (2005): Introduction: Institutional change in advanced political economies, in: Streeck, W./Thelen, K. (Hrsg.): Beyond continuity. Institutional change in advanced political economies, Oxford, S. 1-39

Venn, D. (2012): Eligibility criteria for unemployment benefits: Quantitative indicators for OECD and EU countries, OECD Social, Employment and Migration Working Papers (131)

Werner, D./Ramos Lobato, P./Dietz, M. (2012): Evaluation der Sonderregelung für kurzfristig Beschäftigte in der Arbeitslosenversicherung, IAB-Forschungsbericht (9), Nürnberg

Wissenschaftszentrum Berlin für Sozialforschung (WZB)/Institut für angewandte Sozialwissenschaft (infas) (2006): Evaluation der Maßnahmen zur Umsetzung der Vorschläge der Hartz-Kommission. Modul 1a: Neuausrichtung der Vermittlungsprozesse. Bericht 2006 für das Bundesministerium für Arbeit und Soziales, Berlin/Bonn

\section{AUTOREN}

SILKE BOTHFELD, Prof. Dr., Politikwissenschaftlerin, leitet an der Hochschule Bremen den internationalen Studiengang Politikmanagement. Arbeitsschwerpunkte: International vergleichende Wohlfahrtsstaatsforschung, ArbeitsmarktFamilien- und Gleichstellungspolitik.

silke.bothfeld@hs-bremen.de

PEER ROSENTHAL, M.A. Sozialpolitik, Referent der Geschäftsführung bei der Arbeitnehmerkammer Bremen. Arbeitsschwerpunkte: Arbeitsförderung, soziale Sicherung bei Arbeitslosigkeit.

rosenthal@arbeitnehmerkammer.de 